\title{
Cause-Specific Mortality in Patients with Chronic Kidney Disease and Atrial Fibrillation
}

\author{
Medha Airy ${ }^{a}$ Jesse D. Schold ${ }^{b, d}$ Stacey E. Jolly ${ }^{\mathrm{e}}$ Susana Arrigain ${ }^{c}$ \\ Nisha Bansal ${ }^{f}$ Wolfgang C. Winkelmayer ${ }^{a}$ Joseph V. Nally Jr ${ }^{b}$ \\ Sankar D. Navaneethan ${ }^{\mathrm{a}, \mathrm{g}}$ \\ aSelzman Institute for Kidney Health, Section of Nephrology, Department of Medicine, Baylor College of Medicine, \\ Houston, TX, USA; ${ }^{b}$ Department of Nephrology and Hypertension, Glickman Urological and Kidney Institute, \\ Cleveland Clinic, Cleveland, OH, USA; ' $D$ Department of Quantitative Health Sciences, Cleveland Clinic, Cleveland, \\ $\mathrm{OH}, \mathrm{USA} ;{ }^{\mathrm{d}}$ Center for Populations Health Research, Lerner Research Institute, Cleveland Clinic, Cleveland, OH, USA; \\ ${ }^{\mathrm{e}}$ Medicine Institute, Cleveland Clinic, Cleveland, $\mathrm{OH}, \mathrm{USA} ;{ }^{f}$ Kidney Research Institute, University of Washington, \\ Seattle, WA, USA; ${ }^{9}$ Section of Nephrology, Michael E. DeBakey Veterans Affairs Medical Center, Houston, TX, USA
}

\section{Keywords}

Atrial fibrillation - Cause-specific death .

Chronic kidney disease

\begin{abstract}
Background: Atrial fibrillation (AF) is associated with death in patients with chronic kidney disease (CKD). We examined the associations between AF and cause-specific mortality in a large CKD population. Methods: We included 62,459 patients with estimated glomerular filtration rate $15-59 \mathrm{~mL} /$ min/1.73 $\mathrm{m}^{2}$ (6,639 patients with AF and 55,820 without AF) followed in a large health care system. Outcomes included overall and cause-specific deaths (a) cardiovascular; (b) malignancy; and (c) non-cardiovascular/non-malignancy causes. Cox regression models for overall mortality and separate competing risk models for each major cause of death category were used to evaluate their respective associations
\end{abstract}

\section{KARGER}

(c) 2018 S. Karger AG, Basel

E-Mail karger@karger.com

www.karger.com/ajn with AF. Results: During a median follow-up of 4.1 years, 19,094 patients died; cause of death was known for 18,854 patients. After multivariable adjustment (demographics, comorbidities, relevant laboratory data, medication use, and kidney function), AF was associated with $23 \%$ (95\% Cl $18-$ 29\%) higher risk of all-cause mortality, 45\% (95\% Cl 31-61\%) higher risk of cardiovascular mortality and $13 \%(95 \% \mathrm{Cl}$ 3-22\%) lower risk of malignancy-related mortality. Exclusion of patients with malignancy yielded similar results except for a lack of association between AF and malignancy-related deaths. Results were consistent across various stages of CKD. Conclusions: In a non-dialysis-dependent CKD population, the presence of AF was associated with higher all-cause and cardiovascular mortality. These data suggest that patients with both CKD and AF are at high cardiovascular risk, and thus clinical practice (or trials) should aim at reducing the overall excess cardiovascular mortality (not stroke alone) in patients with AF and CKD.

(c) 2018 S. Karger AG, Basel
Sankar D. Navaneethan, MD, MS, MPH

Selzman Institute for Kidney Health, Section of Nephrology Baylor College of Medicine, 1 Baylor Plaza, Suite 100.37D Houston, TX 77030 (USA)

E-Mail Sankar.navaneethan@bcm.edu 


\section{Background}

Atrial fibrillation (AF) is the most common arrhythmia affecting about $2 \%$ of those aged $<65$ years and $9 \%$ among those aged 65 years or older in the United States [1]. AF is more common in individuals with both nondialysis- and dialysis-dependent chronic kidney disease (CKD) [2-11]. Using the Chronic Renal Insufficiency Cohort study data, Soliman et al. [12] reported that 1 in 5 $\mathrm{CKD}$ patients had AF, a rate similar to that noted in the end-stage renal disease (ESRD) population, and 2-3 times higher than that in the general population. Recent data from the United States Renal Data System report a rising trend in the overall prevalence of AF in CKD (24.5\%), as well as in those with CKD and congestive heart failure, in whom the prevalence increases to over 50\% [13].

$\mathrm{AF}$ is associated with a higher all-cause mortality among patients with ESRD [2,14], as has been shown among patients with non-dialysis-dependent CKD [8, 15-17]. However, less is known about the potential reasons for the increased mortality risk associated with AF. Elucidating the causes of increased mortality in these patients may help guide management to reduce risk. Current strategies to lessen the impact of AF-related outcomes focus mainly on thromboembolism prevention, but patients with AF have high mortality rates from other non-cardiovascular causes as well [18]. Identifying the causes of death would argue for testing targeted interventions beyond using vitamin $\mathrm{K}$ antagonists and direct oral anticoagulants for thromboembolism prevention $[19,20]$. Our study aimed to fill this evidence gap and investigate cause-specific mortality in CKD patients with AF.

\section{Methods}

This analysis was conducted using a pre-existing Electronic Health Record (EHR)-based CKD registry at the Cleveland Clinic. The development and validation of this EHR-based CKD registry has been described in detail elsewhere [21].

\section{Study Population}

Patients who met the following criteria from January 1, 2005 through December 31, 2013 and lived in the State of Ohio were included: (1) had at least 1 face-to-face outpatient encounter with a Cleveland Clinic health care provider and (2) had at least 2 or more estimated glomerular filtration rate (eGFR) $<60 \mathrm{~mL} /$ $\mathrm{min} / 1.73 \mathrm{~m}^{2}$ that were at least 90 days apart (using the CKD Epidemiology Collaboration equation) with the second one being $15-59.9 \mathrm{~mL} / \mathrm{min} / 1.73 \mathrm{~m}^{2}$ [22]. Patients known to have ESRD needing dialysis or renal transplant prior to CKD diagnosis were excluded.

$\mathrm{AF}$ and Causes of Death in CKD

\section{Definitions and Outcome Measures}

Kidney Function

We applied the CKD-Epidemiology Collaboration equation to patients who had 2 outpatient serum creatinine levels between January 1, 2005 and December 31, 2013 to calculate eGFR. All creatinine measurements were performed by the modified kinetic Jaffe reaction using a Hitachi D 2400 Modular Chemistry Analyzer (Roche Diagnostics, Indianapolis, IN) at the Cleveland Clinic laboratory. CKD was defined according to current guidelines as follows: stage 3 CKD (eGFR $30-59 \mathrm{~mL} / \mathrm{min} / 1.73 \mathrm{~m}^{2}$ ) and stage 4 CKD (eGFR 15-29 mL/min/1.73 $\mathrm{m}^{2}$ ). We further categorized stage 3 into CKD stage $3 a\left(e G F R ~ 45-59 \mathrm{~mL} / \mathrm{min} / 1.73 \mathrm{~m}^{2}\right)$ and stage 3b (eGFR 30-44 mL/min/1.73 $\mathrm{m}^{2}$ ).

\section{Atrial Fibrillation}

Prevalent AF was defined as the presence of an International Classification of Diseases (ICD) 9 code 427.31 by providers (at least twice) in the EHR at study entry. We randomly selected 10 patients with $\mathrm{AF}$ and 10 without $\mathrm{AF}$ for chart review validation performed by one of the authors (S.E.J.). Our chart review validation found that $90 \%$ of patients with AF per EMR data extraction had AF and $90 \%$ of patients without AF per EMR did not have AF.

\section{Mortality}

Dates of death were ascertained from the EHR and through linkage of the CKD registry with Ohio Department of Health mortality data, which also provided cause of death information. The reported cause of death was coded according to the ICD-10. We grouped the underlying causes of death as per the National Center for Health Statistics for each coding system, except for some changes as outlined below. We classified deaths into the following 4 major categories: (a) cardiovascular deaths, (b) malignancy, and (c) non-cardiovascular, non-malignancy-related deaths [23]. We defined cardiovascular deaths as deaths due to diseases of the heart, essential hypertension, cerebrovascular disease, atherosclerosis, or other diseases of the circulatory system (ICD-10 codes I00-I78). We defined death due to ischemic heart disease as I20-I25, heart failure as I50, and cerebrovascular disease as I60-I69.

\section{Covariates}

Demographic details were extracted from the EHR. Diabetes mellitus, hypertension, coronary artery disease, congestive heart failure, hyperlipidemia, malignancy, and cerebrovascular disease were defined using pre-specified and validated criteria. Relevant outpatient laboratory values (hemoglobin, albumin) and medication details were obtained from the EHR.

\section{Statistical Analysis}

We compared baseline characteristics between patients with and without AF using chi-square and $t$ tests for categorical and continuous variables respectively. We conducted a logistic regression analysis to identify factors associated with AF adjusting for age, sex, race, diabetes, hypertension, body mass index (BMI), coronary artery disease, congestive heart failure, cerebrovascular disease, peripheral vascular disease, hemoglobin, and eGFR. We tabulated the leading causes of death in those with and without AF. Patients were followed from the second (confirmatory) eGFR measurement $<60 \mathrm{~mL} / \mathrm{min} / 1.73 \mathrm{~m}^{2}$ and censored on December 


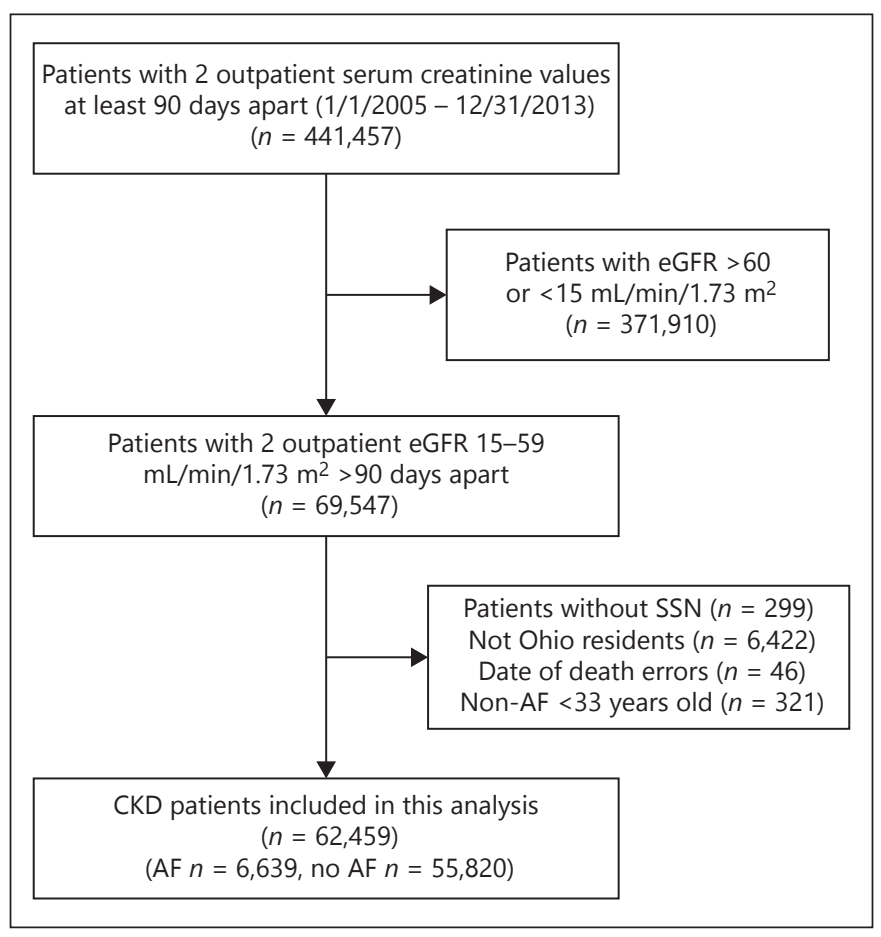

Fig. 1. Flowchart showing how patients were selected to be included in this analysis. AF, atrial fibrillation.

2013. We used unadjusted and adjusted Cox proportional hazards models to evaluate the relationship between AF and all-cause mortality, and competing risks regression analysis to evaluate the relationship between $\mathrm{AF}$ and various causes of death. The mortality models were adjusted for age, sex, race, diabetes, hyperlipidemia, BMI, albumin, hemoglobin, malignancy, hypertension, coronary artery disease, congestive heart failure, cerebrovascular disease, peripheral vascular disease, insurance type, use of Angiotensin converting enzyme inhibitors/angiotensin receptor blockers, beta blocker use, statins use, smoking status, and eGFR. All results were expressed as OR or hazard ratios (HR) with corresponding 95\% CI.

We tested 2-way interactions between AF and age, race, sex, coronary artery disease, and eGFR on overall mortality. We did not account for multiple testing and significance was set at a nominal $p$ value of 0.05 . We performed sensitivity analyses excluding patients with baseline malignancy ( $25 \%$ of the study population) and adjusting for proteinuria among those in whom proteinuria data were available ( $48 \%$ of the study population). The percent of missing information among patients was as follows: $4 \%$ insurance information, $14 \%$ smoking status, $4 \% \mathrm{BMI}, 15 \%$ albumin, and $17 \%$ hemoglobin. We used multiple imputation (SAS proc MI) with the Markov Chain Monte Carlo method and a single chain to impute 5 datasets with complete continuous and binary covariate data in a first step, and then in a second step, we imputed insurance group on each of the 5 datasets using discriminant function analysis. All models were performed on each of the 5 imputed datasets, and parameter estimates were combined using SAS MI analyze.

All analyses were conducted using Linux SAS version 9.4 (SAS Institute, Cary, NC, USA) and graphs were created using R 3.3.2
(The R Foundation for Statistical Computing, Vienna, Austria). The CKD registry and this study were approved by the Cleveland Clinic Institutional Review Board (IRB \#09-015).

\section{Results}

\section{Baseline Patient Characteristics}

Between January 1, 2005 and December 31, 2013, 69,547 patients were included in our CKD registry with eGFR $15-59 \mathrm{~mL} / \mathrm{min} / 1.73 \mathrm{~m}^{2}$. We excluded 7,088 patients because they did not meet the specified inclusion criteria (Fig. 1). Of the remaining 62,459 patients, 6,639 (10.6\%) of patients had AF at cohort entry. The clinical characteristics of the patients with and without AF are presented in Table 1. Patients with AF were older and had a higher proportion of comorbid conditions such as diabetes, hyperlipidemia, coronary artery disease, and congestive heart failure (Table 1). Approximately $80 \%$ of patients with $\mathrm{AF}$ and $9 \%$ of patients without AF were on anticoagulants.

\section{Factors Associated with AF in CKD}

In a multivariable model adjusted for relevant covariates, age, male sex, obesity, hypertension, cardiovascular disease, cerebrovascular disease, congestive heart failure, and stage 4 (versus stage $3 a$ ) CKD were associated with higher odds of having AF (Table 2).

\section{AF and Mortality}

During a median follow-up of 4.1 years, there were 19,094 deaths (AF group: 115.8 deaths per 1,000 years and No AF group: 69.3 deaths per 1,000 years of follow-up). In multivariable analysis, AF was associated with significantly higher hazards of mortality (HR 1.23 95\% CI 1.181.29). Causes of death details were available from the Ohio Department of Health mortality data for 18,854 patients. We excluded the 240 patients whose deaths were only found in the EHR (but not from the Department of Health files) from all cause-specific mortality analyses. Table 3 displays the causes of death overall and by AF. In the models adjusting for relevant covariates, the presence of AF was associated with higher sub-hazards of cardiovascular deaths and lower sub-hazards of malignancy-related deaths (Table 4a). Figure 2 shows the higher subhazards identified for ischemic heart disease, heart failure, and cerebrovascular disease-related deaths among those with AF and CKD. However, in the model in which CKD patients with malignancy at baseline were excluded, the presence of AF was associated only with a higher risk of cardiovascular deaths.
Airy/Schold/Jolly/Arrigain/Bansal/ Winkelmayer/Nally Jr/Navaneethan 
Table 1. Patient characteristics, by presence vs. absence of atrial fibrillation, in the Cleveland clinic CKD Registry

\begin{tabular}{|c|c|c|c|c|}
\hline Factor* & $\mathrm{AF}(n=6639)$ & No $\operatorname{AF}(n=55,820)$ & Overall $(n=62,459)$ & $p$ value \\
\hline Age, years & $76.4 \pm 9.9$ & $71.9 \pm 11.5$ & $72.4 \pm 11.4$ & $<0.001^{\mathrm{a}}$ \\
\hline Gender, male & 54.4 & 42.6 & 43.8 & $<0.001^{\mathrm{c}}$ \\
\hline African American & 8.3 & 13.2 & 12.7 & $<0.001^{\mathrm{c}}$ \\
\hline Smoking & & & & $<0.001^{\mathrm{c}}$ \\
\hline No & 91.3 & 77.3 & 78.8 & \\
\hline Yes & 4.3 & 7.4 & 7.0 & \\
\hline Missing & 4.4 & 15.3 & 14.2 & \\
\hline $\mathrm{BMI}, \mathrm{kg} / \mathrm{m}^{2}$ & $29.1 \pm 6.4$ & $29.5 \pm 6.6$ & $29.5 \pm 6.6$ & $<0.001^{\mathrm{a}}$ \\
\hline BMI categories, $\mathrm{kg} / \mathrm{m}^{2}$ & & & & $<0.001^{\mathrm{c}}$ \\
\hline$<18.5^{\circ}$ & 1.6 & 1.3 & 1.3 & \\
\hline $18.5-24.9$ & 24.3 & 22.3 & 22.5 & \\
\hline $25-29.9$ & 35.5 & 34.0 & 34.1 & \\
\hline $30-34.9$ & 21.0 & 21.3 & 21.3 & \\
\hline $35-39.9$ & 9.0 & 9.8 & 9.7 & \\
\hline$>40$ & 6.0 & 6.9 & 6.8 & \\
\hline Missing & 2.5 & 4.4 & 4.2 & \\
\hline Diabetes & 27.0 & 23.0 & 23.4 & $<0.001^{\mathrm{c}}$ \\
\hline Malignancy & 24.2 & 24.6 & 24.6 & $0.40^{\mathrm{c}}$ \\
\hline Hypertension & 92.8 & 84.0 & 84.9 & $<0.001^{\mathrm{c}}$ \\
\hline Hyperlipidemia & 82.4 & 77.1 & 77.7 & $<0.001^{\mathrm{c}}$ \\
\hline Coronary artery disease & 41.3 & 18.1 & 20.5 & $<0.001^{\mathrm{c}}$ \\
\hline Congestive heart failure & 28.7 & 5.4 & 7.9 & $<0.001^{\mathrm{c}}$ \\
\hline Cerebrovascular disease & 16.4 & 8.3 & 9.2 & $<0.001^{\mathrm{c}}$ \\
\hline Peripheral vascular disease & 4.8 & 3.0 & 3.2 & $<0.001^{\mathrm{c}}$ \\
\hline Liver disease & 5.0 & 5.5 & 5.5 & $0.081^{\mathrm{c}}$ \\
\hline COPD/asthma & 15.4 & 9.7 & 10.3 & $<0.001^{\mathrm{c}}$ \\
\hline ACE-I/ARB & 76.4 & 61.0 & 62.7 & $<0.001^{\mathrm{c}}$ \\
\hline Diuretic & 83.6 & 63.2 & 65.4 & $<0.001^{\mathrm{c}}$ \\
\hline Statin & 66.9 & 55.9 & 57.1 & $<0.001^{\mathrm{c}}$ \\
\hline Beta blocker use & 86.1 & 51.4 & 55.1 & $<0.001^{\mathrm{c}}$ \\
\hline Anticoagulant agents use & 80.0 & 8.8 & 16.4 & $<0.001^{\mathrm{c}}$ \\
\hline Antiplatelet agents use & 18.8 & 14.3 & 14.7 & $<0.001^{\mathrm{c}}$ \\
\hline $\mathrm{eGFR}, \mathrm{mL} / \mathrm{min} / 1.73 \mathrm{~m}^{2}$ & $47.1 \pm 10.3$ & $47.8 \pm 10.3$ & $47.7 \pm 10.3$ & $<0.001^{\mathrm{a}}$ \\
\hline CKD Stage (eGFR range) & & & & $<0.001^{\mathrm{c}}$ \\
\hline Stage $3 a(45-59)$ & 64.7 & 68.0 & 67.6 & \\
\hline Stage $3 b(30-44)$ & 27.4 & 24.3 & 24.6 & \\
\hline Stage $4(15-29)$ & 8.0 & 7.7 & 7.8 & \\
\hline Albumin, g/dL & $4.1 \pm 0.42$ & $4.1 \pm 0.47$ & $4.1 \pm 0.47$ & $0.50^{\mathrm{a}}$ \\
\hline Hemoglobin, mg/dL & $12.8 \pm 1.8$ & $12.8 \pm 1.8$ & $12.8 \pm 1.8$ & $0.049^{\mathrm{a}}$ \\
\hline Proteinuria & 25.4 & 25.5 & 25.5 & $0.85^{\mathrm{c}}$ \\
\hline Insurance & & & & $<0.001^{\mathrm{c}}$ \\
\hline Medicaid & 0.17 & 0.35 & 0.33 & \\
\hline Medicare & 85.9 & 78.4 & 79.2 & \\
\hline Missing & 3.0 & 3.9 & 3.8 & \\
\hline Other & 11.0 & 17.3 & 16.6 & \\
\hline
\end{tabular}

* Data shown as mean \pm SD or column \%.

${ }^{\mathrm{a}} t$ test; ${ }^{\mathrm{c}}$ Chi-square.

$\mathrm{AF}$, atrial fibrillation; BMI, body mass index; COPD, chronic obstructive pulmonary disease; ACE-I, angiotensin converting enzyme inhibitor; ARB, angiotensin II receptor blocker; eGFR, estimated glomerular filtration rate, $\mathrm{CKD}$, chronic kidney disease. 
Table 2. Factors independently associated with atrial fibrillation in the Cleveland clinic CKD registry

\begin{tabular}{lcc}
\hline & OR $(95 \% \mathrm{CI})^{*}$ & $p$ value \\
\hline Age (per 10 years) & $1.46(1.42-1.51)$ & $<0.001$ \\
Gender, male & $1.40(1.32-1.48)$ & $<0.001$ \\
African American race & $0.53(0.48-0.59)$ & $<0.001$ \\
BMI 25-29.9 vs. $18.5-24.9 \mathrm{~kg} / \mathrm{m}^{2}$ & $1.00(0.93-1.08)$ & 0.96 \\
BMI $>30$ vs. $18.5-24.9 \mathrm{~kg} / \mathrm{m}^{2}$ & $1.11(1.03-1.20)$ & 0.01 \\
BMI $<18.5$ vs. $18.5-24.9 \mathrm{~kg} / \mathrm{m}^{2}$ & $1.18(0.95-1.47)$ & 0.13 \\
Coronary artery disease & $1.79(1.68-1.90)$ & $<0.001$ \\
Congestive heart failure & $6.03(5.62-6.48)$ & $<0.001$ \\
Cerebrovascular disease & $1.50(1.38-1.62)$ & $<0.001$ \\
Hypertension & $2.09(1.89-2.32)$ & $<0.001$ \\
Peripheral vascular disease & $0.93(0.81-1.06)$ & 0.27 \\
Hemoglobin Q2 vs. Q1 & $0.96(0.88-1.05)$ & 0.42 \\
Hemoglobin Q3 vs. Q1 & $0.94(0.87-1.03)$ & 0.17 \\
Hemoglobin Q4 vs. Q1 & $1.00(0.91-1.09)$ & 0.91 \\
Diabetes & $0.94(0.88-1.00)$ & 0.06 \\
CKD stage 4 (eGFR $15-29$ vs. $\left.45-59 \mathrm{~mL} / \mathrm{min} / 1.73 \mathrm{~m}^{2}\right)$ & $0.85(0.76-0.95)$ & 0.003 \\
CKD stage 3b (eGFR 30-44 vs. $\left.45-59 \mathrm{~mL} / \mathrm{min} / 1.73 \mathrm{~m}^{2}\right)$ & $1.00(0.93-1.06)$ & 0.89
\end{tabular}

All estimates obtained from MI analyze from the 5 imputed datasets.

* Models adjusted for age, gender, race, diabetes, hypertension, BMI, coronary artery disease, congestive heart failure, cerebrovascular disease, peripheral vascular disease, hemoglobin and eGFR.

Hemoglobin Q1- <11.7 g/dL; Q2- 11.7-12.9 g/dL; Q3- 12.9-14.0 g/dL; Q4- >14.0 g/dL.

BMI, body mass index; CKD, chronic kidney disease.

Table 3. Causes of death, by presence vs. absence of atrial fibrillation, in the Cleveland clinic CKD registry

\begin{tabular}{lccc}
\hline Causes & AF & No AF \\
$(n=2,581)$ & $(n=16,273)$ & $\begin{array}{l}\text { Total } \\
(n=18,854)\end{array}$ \\
\hline All cardiovascular diseases & 49.1 & 32.7 & 35.0 \\
Ischemic heart disease & 23.1 & 16.5 & 17.4 \\
Heart failure & 5.3 & 2.4 & 2.8 \\
Cerebrovascular disease & 4.7 & 4.1 & 4.2 \\
All other cardiovascular diseases & 16.0 & 9.7 & 10.6 \\
Malignant neoplasms & 16.0 & 28.4 & 26.7 \\
Non-cardio non-malignancy & 32.5 & 37.0 & 36.4 \\
Chronic lower respiratory diseases & 4.8 & 4.8 & 4.8 \\
Diabetes mellitus & 3.1 & 4.0 & 3.9 \\
Nephritis, nephrotic syndrome and nephrosis & 3.0 & 2.6 & 2.6 \\
Alzheimer's disease & 1.8 & 2.4 & 2.3 \\
Influenza and pneumonia & 2.4 & 1.9 & 1.9 \\
Septicemia & 1.8 & 1.9 & 1.9 \\
Chronic liver disease and cirrhosis & 0.35 & 1.1 & 1.0 \\
Pneumonitis due to solids and liquids & 1.1 & 0.93 & 0.95 \\
Parkinson's disease & 0.27 & 0.61 & 0.56 \\
All other diseases & 13.9 & 16.8 & 16.4 \\
Non-disease-related deaths & 2.4 & 1.9 & 1.9 \\
\hline
\end{tabular}

Data shown as column \%. AF, atrial fibrillation. 
Table 4.

a Associations of atrial fibrillation with all-cause and cause-specific mortality in the Cleveland clinic CKD registry

\begin{tabular}{|c|c|c|c|c|c|c|}
\hline & $\begin{array}{l}\text { Unadjusted } \\
\text { HR }(95 \% \text { CI })\end{array}$ & $p$ value & $\begin{array}{l}\text { Adjusted* } \\
\text { HR }(95 \% \text { CI })\end{array}$ & $p$ value & $\begin{array}{l}\text { Excluding those with } \\
\text { malignancy adjusted* } \\
\text { HR ( } 95 \% \text { CI) }\end{array}$ & $p$ value \\
\hline \multirow[t]{2}{*}{ All-cause death } & $1.69(1.62-1.76)$ & $<0.001$ & $1.23(1.18-1.29)$ & $<0.001$ & $1.31(1.25-1.39)$ & $<0.001$ \\
\hline & $\begin{array}{l}\text { Unadjusted } \\
\text { SHR (95\% CI) }\end{array}$ & & $\begin{array}{l}\text { Adjusted* } \\
\text { SHR (95\% CI) }\end{array}$ & & $\begin{array}{l}\text { Adjusted* } \\
\text { SHR (95\% CI) }\end{array}$ & \\
\hline Cardiovascular mortality ${ }^{\wedge}$ & $2.40(2.26-2.55)$ & $<0.001$ & $1.45(1.36-1.56)$ & $<0.001$ & $1.48(1.37-1.60)$ & $<0.001$ \\
\hline Malignancy-related mortality ${ }^{\wedge}$ & $0.80(0.73-0.89)$ & $<0.001$ & $0.87(0.78-0.97)$ & 0.016 & $1.01(0.86-1.20)$ & 0.90 \\
\hline $\begin{array}{l}\text { Non-cardio non-malignancy } \\
\text { mortality }\end{array}$ & $1.33(1.23-1.42)$ & $<0.001$ & $1.05(0.96-1.13)$ & 0.28 & $1.02(0.93-1.12)$ & 0.62 \\
\hline
\end{tabular}

* Adjusted for age, sex, race, diabetes, hyperlipidemia, body mass index, albumin, hemoglobin, malignancy (except in analysis excluding patients with baseline malignancy), hypertension, coronary artery disease, congestive heart failure, cerebrovascular disease, peripheral vascular disease, insurance type, ACE/ARB, beta blocker use, statins use, smoking, and eGFR.

Hazard and sub-hazard ratios (HR and SHR) presented in adjusted models were pooled using MIanalyze from 5 multiply imputed datasets.

${ }^{\wedge} n=62,219$ due to some missing cause of death.

b Associations of atrial fibrillation with all-cause and cause-specific mortality among those with proteinuria data $(n=30,143)$

\begin{tabular}{|c|c|c|c|c|}
\hline & $\begin{array}{l}\text { Unadjusted } \\
\text { HR (95\% CI) }\end{array}$ & $p$ value & $\begin{array}{l}\text { Adjusted* } \\
\text { HR }(95 \% \text { CI })\end{array}$ & $p$ value \\
\hline \multirow[t]{2}{*}{ All-cause death } & $1.67(1.57-1.78)$ & $<0.001$ & $1.24(1.16-1.32)$ & $<0.001$ \\
\hline & SHR (95\% CI) & & SHR (95\% CI) & \\
\hline Cardiovascular mortality^ & $2.26(2.06-2.47)$ & $<0.001$ & $1.45(1.31-1.61)$ & $<0.001$ \\
\hline Malignancy-related mortality $\wedge$ & $0.91(0.79-1.04)$ & 0.17 & $0.91(0.78-1.07)$ & 0.24 \\
\hline Non-cardio non-malignancy mortality & $1.33(1.19-1.47)$ & $<0.001$ & $1.08(0.96-1.21)$ & 0.21 \\
\hline \multicolumn{5}{|l|}{ Cardiovascular sub-categories } \\
\hline Ischemic heart disease ${ }^{\wedge}$ & $2.03(1.78-2.32)$ & $<0.001$ & $1.28(1.10-1.48)$ & 0.001 \\
\hline Heart failure ${ }^{\wedge}$ & $2.63(1.94-3.56)$ & $<0.001$ & $1.22(0.86-1.73)$ & 0.22 \\
\hline Cerebrovascular disease $^{\wedge}$ & $1.73(1.30-2.29)$ & $<0.001$ & $1.30(0.95-1.78)$ & 0.10 \\
\hline Other cardiovascular diseases ${ }^{\wedge}$ & $2.35(2.00-2.76)$ & $<0.001$ & $1.69(1.40-2.03)$ & $<0.001$ \\
\hline
\end{tabular}

* Adjusted for age, sex, race, diabetes, hyperlipidemia, body mass index, albumin, hemoglobin, malignancy (except in analysis excluding patients with baseline malignancy), hypertension, coronary artery disease, congestive heart failure, cerebrovascular disease, peripheral vascular disease, insurance type, ACE/ARB, beta blocker use, statins use, smoking, eGFR and proteinuria.

Hazard and sub-hazard ratios presented in adjusted models were pooled using MIanalyze from 5 multiply imputed datasets.

${ }^{\wedge} n=30,033$ due to some missing cause of death.

\section{Effect Modification}

The multiplicative interaction between AF and age on overall mortality was significant $(p=0.02)$ suggesting that the risk associated with $\mathrm{AF}$ was stronger among younger patients (HR 1.39, 95\% CI 1.22-1.60 for patients <65 years vs. HR 1.30, 95\% CI 1.24-1.36 for $\geq 65$ years). The interaction between $\mathrm{AF}$ and sex on overall mortality was also significant $(p=0.04)$ suggesting that the hazard associated with AF was significant for both sexes but stronger among women (HR 1.29, 95\%
CI 1.21-1.38 for women vs. HR 1.18, 95\% CI 1.12-1.26 for men). The interactions between AF and race, eGFR and pre-existing cardiovascular disease were not nominally significant.

\section{Sensitivity Analysis}

Table $4 \mathrm{a}$ also shows the results of the sensitivity analysis excluding patients with baseline malignancy. These results were similar for cardiovascular and non-cardiovascular non-malignancy mortality. When excluding pa- 


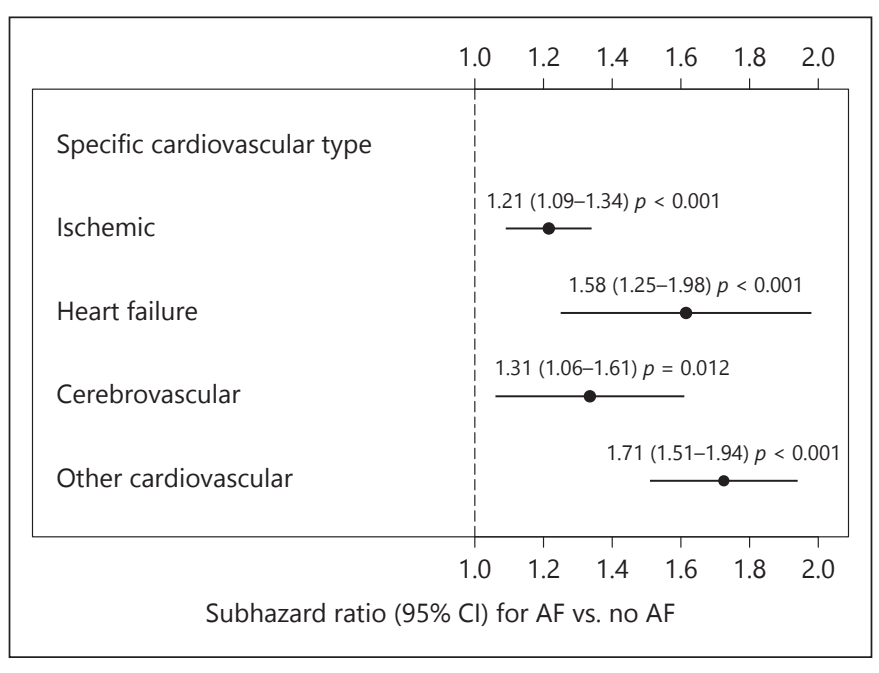

Fig. 2. Associations of AF with specific type of cardiovascular deaths in those with CKD. Model adjusted for age, sex, race, diabetes, hyperlipidemia, body mass index (BMI), albumin, hemoglobin, malignancy (except in analysis excluding patients with baseline malignancy), hypertension, coronary artery disease, congestive heart failure, cerebrovascular disease, peripheral vascular disease, insurance type, use of ACE/angiotensin receptor blocker (ARB), beta blocker use, statins use, smoking status, and eGFR.

tients with baseline malignancy, malignancy-related mortality was not significantly different between those with and without AF. The analysis adjusted for proteinuria yielded results, similar to the primary analysis (Table 4b).

\section{Discussion}

In a well-described cohort of patients with CKD who were followed in a usual health care setting, prevalent AF was noted among $11 \%$ of the study population. Various demographic variables, comorbid conditions and stage $4 \mathrm{CKD}$ were associated with higher odds of having AF. We also report an increased risk of all-cause death among those with AF and CKD. These results are consistent with prior reports noting similar associations $[8,15-17]$. We were specifically interested in cause-specific mortality in this population with both $\mathrm{AF}$ and CKD and found that cardiovascular deaths accounted for approximately half of the deaths. Even though there was a lower risk of malignancy-related death among those with AF and CKD, such an association was no longer present when patients with pre-existing malignancy were excluded. Cumulatively, these data provide novel insights about etiologies for death among this high-risk population with concurrent $\mathrm{CKD}$ and $\mathrm{AF}$.

In the general population, associations between $\mathrm{AF}$ and various cause-specific deaths have been investigated. Fauchier et al. [24] reported that among 8,962 patients with incident AF, majority of deaths during follow-up were related to cardiovascular disease (54\%), and heart failure (29\%) was specifically noted to be the most common cause of death. Notably, despite the high risk of stroke with AF, only 7\% died from stroke [24]. GomezOutez et al. [25] pooled data from 4 clinical trials representing 71,683 patients with AF. They noted that cardiac deaths accounted for $46 \%$ of all deaths, and stroke-related deaths accounted for $11.3 \%$ of the total mortality [25]. Such comprehensive assessment of cause-specific death is lacking for the large and important CKD substratum within the population with AF. Similar to the general population, cardiovascular deaths accounted for a majority of deaths (49\%) in our analysis. Specifically, ischemic heart disease, heart failure, and cerebrovascular disease were associated with 21,58 , and $31 \%$ increased sub-hazards of death, respectively, in these patients with both AF and CKD. Literature examining the associations of cardiovascular events with increased mortality in CKD patients with AF is quite limited. In a single-center study, Nakagawa and colleagues reported that combined eGFR and $\mathrm{CHADS}_{2}$ scoring was a predictor for cardiovascular events and mortality in patients with AF [26]. However, data on non-CVD causes and subtypes of CVD death are sparse for patients with CKD and AF. Despite the fact that patients with $\mathrm{AF}$ and $\mathrm{CKD}$ were older and with higher comorbidity burden (Table 1), the data from our study highlights the poor prognosis associated with $\mathrm{AF}$ and may help prioritize CVD or other treatment in this highrisk population.

In those with $\mathrm{AF}$, the presence of $\mathrm{CKD}$ is associated with stroke and thromboembolic events and increased mortality [15, 17, 27-31]. Olesen et al. studied 132,372 patients from the Danish general population and noted an increased risk of stroke or systemic thromboembolism in patients with CKD (HR 1.49, 95\% CI 1.38-1.59) [28]. Similar results have also been noted in other studies with some reporting higher risk than others and the difference is attributed to the discrepancies in the inclusion criteria $[2,15,17,30-32]$. Our study results further reaffirm the associations between AF and cerebrovascular disease-related deaths in CKD. However, due to the nature of our study (death certificate derived data), we were not able to differentiate ischemic versus hemorrhagic cerebrovascular events in our study. Further studies from longitudinal
Airy/Schold/Jolly/Arrigain/Bansal/ Winkelmayer/Nally Jr/Navaneethan 
cohorts of kidney population could inform us about the risk estimates for various types of cerebrovascular deaths in CKD.

Much has been debated about the need for oral anticoagulation in patients with $\mathrm{AF}$ and though it is recommended for the general population based on $\mathrm{CHA}_{2} \mathrm{DS}_{2^{-}}$ VASc and HAS-BLED score combination, no conclusive evidence exists to support their use in advanced CKD (CKD $3 \mathrm{~b}$ and higher) due to lack of representation of these patients in the pivotal anticoagulation trials and equivocal results from the existing observational data [19, 27, 28, 33-36]. Data from the Alberta Kidney Disease Network reported a lower risk of a composite outcome of all-cause death, ischemic stroke, and transient ischemic attack with warfarin therapy initiation among CKD patients with AF (adjusted HR from 0.54 to 0.64 for different CKD stages) and no increased risk of major bleeding except for those with eGFR $60-89 \mathrm{~mL} / \mathrm{min} / 1.73 \mathrm{~m}^{2}$ (HR 1.36, 95\% CI 1.13-1.64) [35]. Carrero et al. [37] reported that treatment with warfarin was associated with a lower 1-year risk of outcomes (death, MI, and ischemic stroke) without a higher risk of bleeding in those with acute myocardial infarction and AF and the association was not related to the severity of concurrent CKD. Observational studies are prone to bias and hence further dedicated clinical trials addressing this key issue in CKD are warranted.

Studies have reported sex-specific differences in the epidemiology and clinical outcomes of AF in the general population [38]. Multiple mechanisms have been proposed to address these differences including the role of estrogen replacement therapy [39]. While some suggested a lower risk of AF with hormone replacement therapy, others have refuted those findings [40, 41]. Even though modest, in CKD population, we noted sex-specific differences in the associations between AF and mortality with a stronger association in women than in men. Additional studies addressing the mechanisms that could explain these differences and whether there exist any differential treatment responses in patients with CKD are needed. In the general population, recent data suggest that African Americans with AF sustain higher rates of stroke, heart failure, and mortality than whites [42]. However, we did not find racial differences (based on the test of interaction) on outcome among those with CKD and AF.

Among the strengths of this study are the use of a large diverse clinical population of patients with stage 3 and 4 $\mathrm{CKD}$ and the availability of information on all-cause and cause-specific deaths. However, there are several limitations that merit discussion. These include the observational nature of this analysis limiting any causal interpre- tation. Further, these data were derived from a single large health system, and hence, the results may not be generalizable to other health care systems and CKD populations. Even though we validated the diagnosis of AF in our chart review, we were not able to differentiate nonvalvular and valvular AF, which could have different implications. While a large proportion of the study population obtained a majority of their care within the Cleveland Clinic health care system, we cannot account for care that may have occurred outside of the Cleveland Clinic system. We also lacked comprehensive data to calculate stroke $\left(\mathrm{CHA}_{2} \mathrm{DS}_{2}-\mathrm{VASc}\right)$ and bleeding (HAS-BLED) risk scores or to compute the illness severity score/index. We also lacked comprehensive medication data to conduct additional analyses affecting the outcomes. The difference in exposure to the medications including anti-arrhythmic and anti-coagulants could possibly explain the lower risk of $\mathrm{AF}$ in older patients along with the competing risk from death due to other causes. Due to the nature of the study, we also lacked echocardiogram data. Further it is unclear if the inclusion of patients who did not receive treatment but potentially could have benefitted from anticoagulation could have influenced the results. While we obtained cause-specific death data, this information was derived from death certificates, which is subject to limitations. However, we had previously validated the reliability of the cause-specific death data for other, previous studies $[43,44]$.

In conclusion, we found an increased risk of all-cause mortality with AF in those with CKD and this higher risk was primarily related to cardiovascular disease-related deaths, specifically ischemic heart disease, congestive heart failure, and cerebrovascular disease. Our data reinforce that patients with both CKD and AF are at high cardiovascular risk, and not just from ischemic stroke, thus suggesting that clinical practice (or trials) should aim at reducing the overall excess cardiovascular mortality in patients with $\mathrm{AF}$ and $\mathrm{CKD}$.

\section{Disclosure Statement}

Authors report no conflicts with respect to the contents of this manuscript.

\section{Funding Source}

S.D.N. is supported by a grant from the National Institutes of Health (NIDDK-R01DK101500). The content is solely the responsibility of the authors and does not necessarily represent the official 
views of the NIH. NIH did not have any role in study design; collection, analysis, and interpretation of data; writing the report; and the decision to submit the report for publication. The creation of the CCF CKD registry was funded by an unrestricted grant from Amgen, Inc to the Department of Nephrology and Hypertension Research and Education Fund.

\section{Previous Presentations}

The results of this study were presented as a poster at the 2017 Kidney Week of the American Society of Nephrology in New Orleans, LA. Otherwise, the results presented in this paper have not been published previously in whole or part, except in the abstract format.

\section{References}

1 Writing Group Members, Mozaffarian D, Benjamin EJ, et al: Heart disease and stroke statistics-2016 update: A report from the american heart association. Circulation 2016; 133:e38-e360.

2 Abbott KC, Trespalacios FC, Taylor AJ, Agodoa LY: Atrial fibrillation in chronic dialysis patients in the united states: risk factors for hospitalization and mortality. BMC Nephrol 2003;4:1.

3 Winkelmayer WC, Patrick AR, Liu J, Brookhart MA, Setoguchi S: The increasing prevalence of atrial fibrillation among hemodialysis patients. J Am Soc Nephrol 2011;22: 349-357.

4 Alonso A, Lopez FL, Matsushita K, et al: Chronic kidney disease is associated with the incidence of atrial fibrillation: The atherosclerosis risk in communities (ARIC) study. Circulation 2011;123:2946-2953.

5 Baber U, Howard VJ, Halperin JL, et al: Association of chronic kidney disease with atrial fibrillation among adults in the united states: REasons for geographic and racial differences in stroke (REGARDS) study. Circ Arrhythm Electrophysiol 2011;4:26-32.

6 Iguchi Y, Kimura K, Kobayashi K, et al: Relation of atrial fibrillation to glomerular filtration rate. Am J Cardiol 2008;102:1056-1059.

7 Horio T, Iwashima Y, Kamide K, et al: Chronic kidney disease as an independent risk factor for new-onset atrial fibrillation in hypertensive patients. J Hypertens 2010;28:1738-1744.

8 Nelson SE, Shroff GR, Li S, Herzog CA: Impact of chronic kidney disease on risk of incident atrial fibrillation and subsequent survival in medicare patients. J Am Heart Assoc 2012;1:e002097.

9 Ohyama Y, Imai M, Kurabayashi M: Estimated glomerular filtration rate and proteinuria are separately and independently associated with the prevalence of atrial fibrillation in general population. PLoS One 2013;8:e79717.

10 Watanabe H, Watanabe T, Sasaki S, Nagai K, Roden DM, Aizawa Y: Close bidirectional relationship between chronic kidney disease and atrial fibrillation: the niigata preventive medicine study. Am Heart J 2009;158:629636.

11 Ananthapanyasut W, Napan S, Rudolph EH, et al: Prevalence of atrial fibrillation and its predictors in nondialysis patients with chronic kidney disease. Clin J Am Soc Nephrol 2010;5:173-181.
12 Soliman EZ, Prineas RJ, Go AS, et al: Chronic kidney disease and prevalent atrial fibrillation: the chronic renal insufficiency cohort (CRIC). Am Heart J 2010;159:1102-1107.

13 USRDS ADR: USRDS report 2016, 2016.

14 Genovesi S, Vincenti A, Rossi E, et al: Atrial fibrillation and morbidity and mortality in a cohort of long-term hemodialysis patients. Am J Kidney Dis 2008;51:255-262.

15 Banerjee A, Fauchier L, Vourc'h P, et al: A prospective study of estimated glomerular filtration rate and outcomes in patients with atrial fibrillation: the loire valley atrial fibrillation project. Chest 2014;145:1370-1382.

16 Bansal N, Fan D, Hsu CY, Ordonez JD, Go AS: Incident atrial fibrillation and risk of death in adults with chronic kidney disease. J Am Heart Assoc 2014;3:e01303.

17 Guo Y, Wang H, Zhao X, et al: Sequential changes in renal function and the risk of stroke and death in patients with atrial fibrillation. Int J Cardiol 2013;168:4678-4684.

18 Marijon E, Le Heuzey JY, Connolly S, et al: Causes of death and influencing factors in patients with atrial fibrillation: a competing-risk analysis from the randomized evaluation of long-term anticoagulant therapy study. Circulation 2013;128:2192-2201.

19 Bansal VK, Herzog CA, Sarnak MJ, et al: Oral anticoagulants to prevent stroke in nonvalvular atrial fibrillation in patients with CKD stage 5D: An NKF-KDOQI controversies report. Am J Kidney Dis 2017;70:859-868.

20 Kimachi M, Furukawa TA, Kimachi K, Goto Y, Fukuma S, Fukuhara S: Direct oral anticoagulants versus warfarin for preventing stroke and systemic embolic events among atrial fibrillation patients with chronic kidney disease. Cochrane Database Syst Rev 2017;11: CD011373.

21 Navaneethan SD, Jolly SE, Schold JD, et al: Development and validation of an electronic health record-based chronic kidney disease registry. Clin J Am Soc Nephrol 2011;6:4049.

22 Levey AS, Stevens LA, Schmid CH, et al: A new equation to estimate glomerular filtration rate. Ann Intern Med 2009;150:604-612.

23 Navaneethan SD, Schold JD, Arrigain S, Jolly SE, Nally JV Jr: Cause-specific deaths in nondialysis-dependent CKD. J Am Soc Nephrol 2015;26:2512-2520.

24 Fauchier L, Villejoubert O, Clementy N, et al: Causes of death and influencing factors in pa- tients with atrial fibrillation. Am J Med 2016; 129:1278-1287.

25 Gomez-Outes A, Lagunar-Ruiz J, TerleiraFernandez AI, Calvo-Rojas G, Suarez-Gea ML, Vargas-Castrillon E: Causes of death in anticoagulated patients with atrial fibrillation. J Am Coll Cardiol 2016;68:2508-2521.

26 Nakagawa K, Hirai T, Takashima S, et al: Chronic kidney disease and CHADS(2) score independently predict cardiovascular events and mortality in patients with nonvalvular atrial fibrillation. Am J Cardiol 2011;107:912-916.

27 Lau YC, Proietti M, Guiducci E, Blann AD, Lip GYH: Atrial fibrillation and thromboembolism in patients with chronic kidney disease. J Am Coll Cardiol 2016;68:1452-1464.

28 Olesen JB, Lip GY, Kamper AL, et al: Stroke and bleeding in atrial fibrillation with chronic kidney disease. N Engl J Med 2012;367:625635.

29 Piccini JP, Stevens SR, Chang Y, et al: Renal dysfunction as a predictor of stroke and systemic embolism in patients with nonvalvular atrial fibrillation: Validation of the $\mathrm{R}(2)$ CHADS(2) index in the ROCKET AF (rivaroxaban once-daily, oral, direct factor Xa inhibition compared with vitamin $\mathrm{K}$ antagonism for prevention of stroke and embolism trial in atrial fibrillation) and ATRIA (AnTicoagulation and risk factors in atrial fibrillation) study cohorts. Circulation 2013;127:224-232.

30 Providencia R, Marijon E, Boveda S, et al: Meta-analysis of the influence of chronic kidney disease on the risk of thromboembolism among patients with nonvalvular atrial fibrillation. Am J Cardiol 2014;114:646-653.

31 Wetmore JB, Ellerbeck EF, Mahnken JD, et al: Atrial fibrillation and risk of stroke in dialysis patients. Ann Epidemiol 2013;23:112-118.

32 Zimmerman D, Sood MM, Rigatto C, Holden RM, Hiremath S, Clase CM: Systematic review and meta-analysis of incidence, prevalence and outcomes of atrial fibrillation in patients on dialysis. Nephrol Dial Transplant 2012;27:3816-3822.

33 Bansal N: The debate on warfarin use in dialysis patients with atrial fibrillation: More fuel for the fire. Am J Kidney Dis 2014;64: 677-680.

34 Winkelmayer WC, Turakhia MP: Warfarin treatment in patients with atrial fibrillation and advanced chronic kidney disease: sins of omission or commission? Jama 2014;311: 913-915. 
35 Jun M, James MT, Ma Z, et al: Warfarin initiation, atrial fibrillation, and kidney function: comparative effectiveness and safety of warfarin in older adults with newly diagnosed atrial fibrillation. Am J Kidney Dis 2017;69: 734-743.

36 Hart RG, Pearce LA, Asinger RW, Herzog CA: Warfarin in atrial fibrillation patients with moderate chronic kidney disease. Clin J Am Soc Nephrol 2011;6:2599-2604.

37 Carrero JJ, Evans M, Szummer K, et al: Warfarin, kidney dysfunction, and outcomes following acute myocardial infarction in patients with atrial fibrillation. JAMA 2014;311: 919-928.
38 Gillis AM: Atrial fibrillation and ventricular arrhythmias: Sex differences in electrophysiology, epidemiology, clinical presentation, and clinical outcomes. Circulation 2017;135: 593-608

39 Ko D, Rahman F, Martins MA, et al: Atrial fibrillation in women: treatment. Nat Rev Cardiol 2017;14:113-124.

40 Perez MV, Wang PJ, Larson JC, et al: Effects of postmenopausal hormone therapy on incident atrial fibrillation: The women's health initiative randomized controlled trials. Circ Arrhythm Electrophysiol 2012;5:11081116.

41 Bretler DM, Hansen PR, Lindhardsen J, et al: Hormone replacement therapy and risk of new-onset atrial fibrillation after myocardial infarction - a nationwide cohort study. PLoS One 2012;7:e51580.
42 Magnani JW, Norby FL, Agarwal SK, et al: Racial differences in atrial fibrillation-related cardiovascular disease and mortality: the atherosclerosis risk in communities (ARIC) study. JAMA Cardiol 2016;1:433-441.

43 Navaneethan SD, Schold JD, Jolly SE, et al Blood pressure parameters are associated with all-cause and cause-specific mortality in chronic kidney disease. Kidney Int 2017;92: 1272-1281.

44 Navaneethan SD, Schold JD, Jolly SE, Arrigain S, Winkelmayer WC, Nally JV Jr: Diabetes control and the risks of ESRD and mortality in patients with CKD. Am J Kidney Dis 2017;70:191-198. 